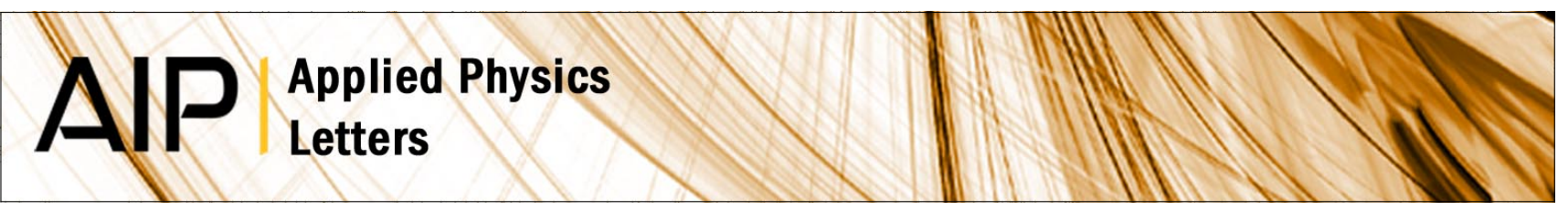

\title{
Dual band terahertz waveguiding on a planar metal surface patterned with annular holes
}

C. R. Williams, M. Misra, S. R. Andrews, S. A. Maier, S. Carretero-Palacios et al.

Citation: Appl. Phys. Lett. 96, 011101 (2010); doi: 10.1063/1.3276545

View online: http://dx.doi.org/10.1063/1.3276545

View Table of Contents: http://apl.aip.org/resource/1/APPLAB/v96/i1

Published by the American Institute of Physics.

Additional information on Appl. Phys. Lett.

Journal Homepage: http://apl.aip.org/

Journal Information: http://apl.aip.org/about/about_the_journal

Top downloads: http://apl.aip.org/features/most_downloaded

Information for Authors: http://apl.aip.org/authors

\section{ADVERTISEMENT}

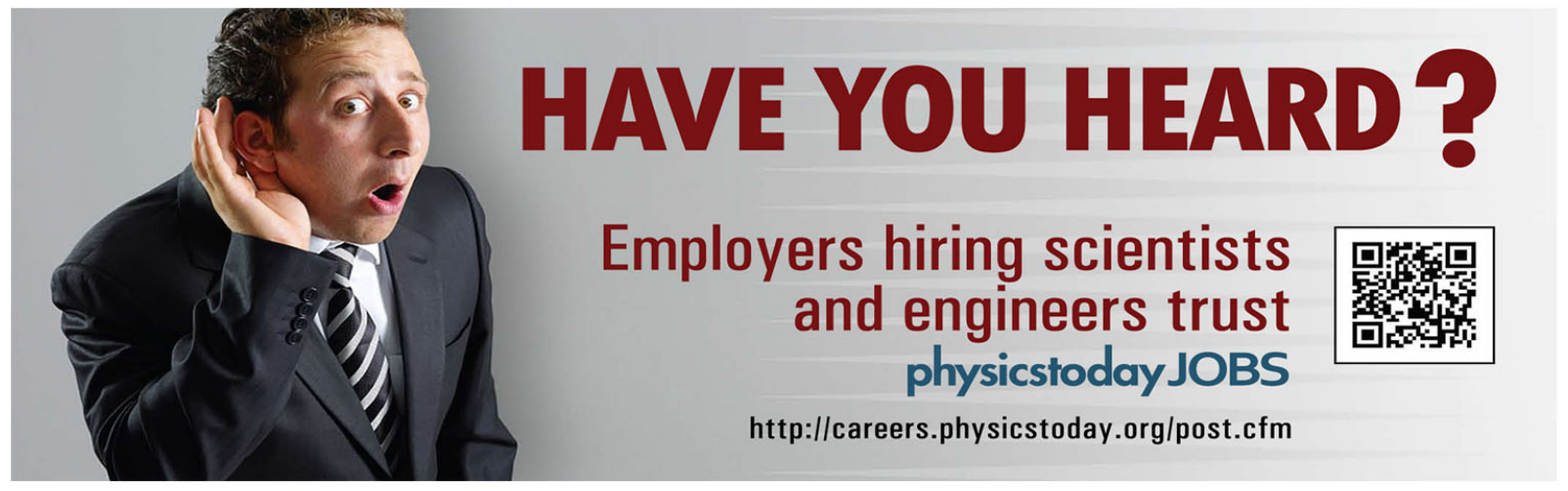




\title{
Dual band terahertz waveguiding on a planar metal surface patterned with annular holes
}

\author{
C. R. Williams, ${ }_{3}^{1}$ M. Misra, ${ }^{1}$ S. R. Andrews, ${ }^{1, a)}$ S. A. Maier ${ }_{3}^{2}$ S. Carretero-Palacios, ${ }^{3}$ \\ S. G. Rodrigo, ${ }^{3}$ F. J. Garcia-Vidal, ${ }^{4}$ and L. Martin-Moreno ${ }^{3}$ \\ ${ }_{1}^{1}$ Department of Physics, University of Bath, Bath BA2 7AY, United Kingdom \\ ${ }^{2}$ Department of Physics, Experimental Solid State Group, Imperial College London, London SW7 2AZ, \\ United Kingdom \\ ${ }^{3}$ Instituto de Ciencia de Materiales de Aragaon (ICMA) and Departamento de Física de la Materia \\ Condensada, CSIC-Universidad de Zaragoza, E-5009 Zaragoza, Spain \\ ${ }^{4}$ Departamento de Física Teórica de la Materia Condensada, Universidad Autónoma de Madrid, \\ E-28049 Madrid, Spain
}

(Received 28 October 2009; accepted 25 November 2009; published online 4 January 2010)

\begin{abstract}
We report studies of the guiding of terahertz radiation on a copper surface textured with an array of blind annular holes. The structure supports two tightly bound surface plasmon-polaritonlike electromagnetic waves associated with $\mathrm{TE}_{11}$ and $\mathrm{TEM}$ coaxial waveguide modes. The $\mathrm{TE}_{11}$-like surface mode has a cutoff determined by the array period while the TEM mode can be arranged to have a lower frequency cut-off by adjusting the hole depth. The ability to guide two modes in independently variable bands could be useful in quantitative chemical sensing. () 2010 American Institute of Physics. [doi:10.1063/1.3276545]
\end{abstract}

The terahertz $(\mathrm{THz})$ frequency spectrum is conventionally taken to be between about 0.1 and $10 \mathrm{THz}$ and lies between the domains of microwave electronics and mid infrared optics. Developments in this band are strongly motivated by applications in fundamental science and the quest for higher bandwidth signal processing and new imaging and sensing modalities. ${ }^{1}$ Examples of current or emerging applications include the dielectric characterization of materials, ${ }^{2}$ medical imaging, ${ }^{3}$ pharmaceutical quality control, $^{4}$ communications, ${ }^{5}$ security screening, ${ }^{6}$ and chemical and biochemical sensing. ${ }^{7}$ Potential sensing applications, for example in label free genetic testing, often involve small volumes of material. In this case it is desirable to enhance the sensitivity by confining the probing radiation in the region of the sample on a wavelength scale or better in at least one dimension. Other important considerations are whether guiding can be achieved over a frequency range sufficient for spectroscopic discrimination and whether there is easy access to the guided mode. Various approaches to sensing have been suggested, including the use of stripline resonators, ${ }^{8}$ parallel plate waveguides, ${ }^{9}$ attenuated total internal reflection, ${ }^{10}$ and plasmonic metamaterials. ${ }^{11}$

Plasmonic metamaterials are artificial metal-dielectric composites structured on a subwavelength scale. They can support tightly bound electromagnetic surface waves at $\mathrm{THz}$ frequencies that mimic the behavior of surface plasmon polaritons (SPPs) in the visible but with effective plasma frequencies determined by surface geometry rather than the properties of the specific metal. ${ }^{12}$ The out-of-plane extent of these "spoof" surface plasmon polaritons (which we refer to as SSPPs hereafter) is determined by their dispersion which can be readily engineered. Among the simplest plasmonic metamaterials are metal surfaces decorated with square or circular holes. The holes can be considered as waveguides and have a cutoff frequency, equivalent to the effective

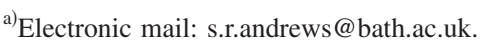

plasma frequency, below which only evanescent fields exist on the metal side of the interface. In the case of a periodic array of air filled holes, the effective plasma frequency lies above the band edge and cannot be accessed. The periodicity then determines the cutoff frequency below which SSPPs can propagate. Strong spatial confinement of SSPPs perpendicular to the surface is still achieved close to the band edge because of the way in which the dispersion curve is bent below the light line by coherent scattering. Recently, we reported direct observation of wavelength scale confinement over an octave of $\mathrm{THz}$ frequency due to this effect on flat metal surfaces decorated with periodic arrays of blind, square holes. ${ }^{11}$ While effective, this approach does not lend itself to guiding in multiple frequency bands or allow access to the subwavelength-scale field confinement that is possible close to the effective plasma frequency. These limitations can be addressed using different hole geometries. In this letter we describe experiments and numerical simulations designed to study dual band THz SSPP propagation on a copper surface textured with an array of blind annular holes. In the optical and near infrared, SSPPs on arrays of nanoscale coaxial apertures play a part in the extraordinary near infrared optical transmission through such structures. ${ }^{13,14}$

The metamaterial investigated is illustrated in Fig. 1(a). The period is $\Lambda=80 \mu \mathrm{m}$ and the hole depth, $h$, is approximately $60 \mu \mathrm{m}$. The inner and outer diameters of each annulus are $a_{\text {in }}=20 \mu \mathrm{m}$ and $a_{\text {out }}=60 \mu \mathrm{m}$. The structure was made using photolithography with SU-8-50 resist ${ }^{15}$ followed by sputter coating with $3 \mu \mathrm{m}$ of copper and diced into $8 \mathrm{~mm} \times 42 \mathrm{~mm}$ rectangular samples. From cross sectional scanning electron microscope images such as that in Fig. 1(b), the coating thickness on the side-walls, where the metal is thinnest, is estimated to be in the range 0.5 to $1 \mu \mathrm{m}$ which is an order of magnitude larger than the skin depth at $1 \mathrm{THz}$.

The two lowest frequency modes of an infinitely long coaxial waveguide are a cylindrically symmetric TEM mode which has no cutoff and a $\mathrm{TE}_{11}$ mode with cutoff determined by the average radius. For coaxial grooves of finite depth $h$, 

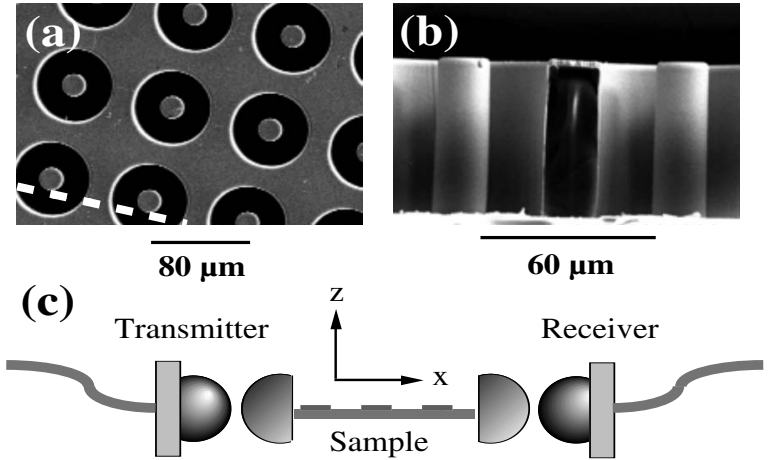

FIG. 1. (a) Electron micrograph of the metamaterial top surface. (b) Cross section along the dotted line in (a). The white area at the bottom of (b) is the pyrex substrate. (c) The experimental geometry.

the TEM surface mode cuts off at $f_{c} \sim c /(4 h)$ and the $\mathrm{TE}_{11}$ mode at $f_{c} \sim 2 c /\left[\pi\left(a_{\mathrm{in}}+a_{\text {out }}\right)\right] .{ }^{16}$ Our periodic structure of annular grooves supports SSPPs with local field patterns similar to those of the TEM and $\mathrm{TE}_{11}$ modes of the infinite guide, as shown in the insets to Fig. 2. The dispersion curves of these TEM-like and $\mathrm{TE}_{11}$-like SSPP modes were calculated in the perfect conductor approximation using finite difference time domain (FDTD) simulations and, with very similar results, by an analytical coupled mode method $(\mathrm{CMM})$ and are shown in Fig. 2. In the CMM, ${ }^{17}$ diffraction was included to arbitrary order but only the TEM and $\mathrm{TE}_{11}$ modes were considered, in order to show that these make the dominant contribution. TM and higher order TE modes have frequencies above $7 \mathrm{THz}$ and can be neglected. The TEMlike spoof plasma frequency is determined by the hole depth and lies at $f_{c}=1.06 \mathrm{THz}$ for $h=60 \mu \mathrm{m}$. It can be varied between roughly 1.7 and $0.3 \mathrm{THz}$ by selecting $h$ in the range 30-100 $\mu \mathrm{m}$. The $\mathrm{TE}_{11}$ waveguide cutoff in our structure lies near $5 \mathrm{THz}$ and is above the free space frequency at the zone boundary so that the effective $\mathrm{TE}_{11}$-like cut off is instead determined by the period of the structure and has a frequency $f_{c}^{*}=1.8 \mathrm{THz}$.

Time domain $\mathrm{THz}$ spectroscopy using a photoconductive transmitter and receiver combination ${ }^{11}$ was performed on the structure in the geometry shown in Fig. 1(c). End-fire coupling of the $s$-polarized incident radiation to guided modes on the metamaterial was achieved using high resistivity silicon hyper-hemispherical collimating lenses in conjunction

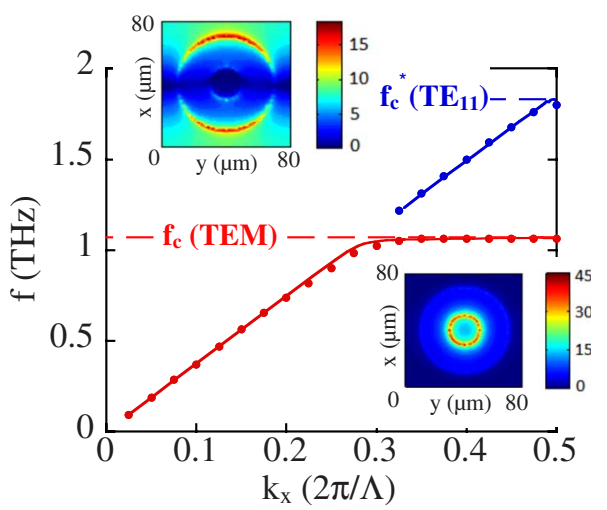

FIG. 2. (Color online) Metamaterial dispersion curves calculated using FDTD simulations (points) and the CWA (continuous curves). $k_{x}$ is the wavevector parallel to the surface in the direction of propagation. The insets show maps of the absolute electric field strength $1 \mu \mathrm{m}$ above the surface of a unit cell at the TEM (upper left) and $\mathrm{TE}_{11}$-like (lower right) SSPP cutoff frequencies.
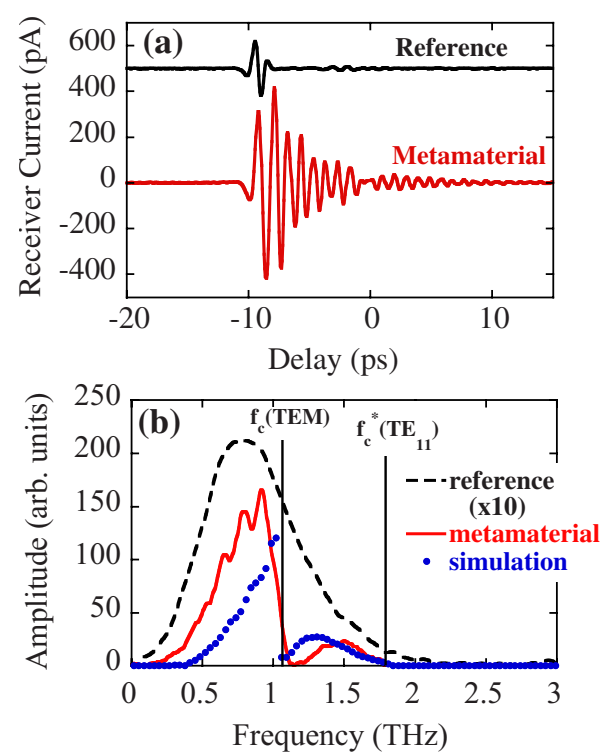

FIG. 3. (Color online) (a) Experimental time domain trace showing SSPP propagation on the metamaterial and a reference trace in air. (b) Spectra corresponding to the traces in (a) and the spectrum calculated using the finite integration technique $100 \mu \mathrm{m}$ above the surface. The vertical lines show the positions of the cut-off frequencies for the TEM and $\mathrm{TE}_{11}$-like SSPPs obtained using the FDTD technique.

with silicon planocylindrical focusing lenses. The flat faces of the cylindrical lenses were spaced from the sample edges by $50 \mu \mathrm{m}$ thick Mylar films and the optical axis was arranged to lie about $100 \mu \mathrm{m}$ above the sample surface. Using a scanning photoconductive antenna, the beam focus at the lens face on the in-coupling side was found to have a shortaxis full width at half maximum amplitude of $200 \mu \mathrm{m}$ at the lens surface. We find that the end-fire coupling geometry is about five times more efficient than the broadband technique based on obliquely illuminated apertures used previously. ${ }^{11,18}$

Figure 3(a) shows time domain data for the metamaterial, together with a reference signal recorded to show the system frequency response and to calibrate the spectral phase. The reference was obtained by removing the sample while keeping the optics fixed in position. The metamaterial trace shows dispersion and beating which are signatures of multiple guided SSPP modes. These are clearly shown in the corresponding spectra in Fig. 3(b). The small oscillations evident below $1 \mathrm{THz}$ are a reflection artifact. The vertical dashed lines in Fig. 3(b) show the frequencies of the TEM and $\mathrm{TE}_{11}$ SSPP cut-offs evaluated in FDTD simulations. In time domain simulations using the finite integration technique (FIT) (Ref. 19) and sufficiently broadband illumination we find that roughly equal energy is coupled into each mode. The lower amplitude of the $\mathrm{TE}_{11}$ mode relative to that of the TEM mode in Fig. 3(b) is largely a result of the frequency response of the THz system which is described by the reference spectrum in Fig. 3(b). If the input spectrum in the FIT simulation is matched to that of the reference then we obtain reasonable agreement between measured and calculated spectra, as shown in Fig. 3(b), which verifies that guiding takes place in both the $\mathrm{TE}_{11}$ and TEM-like surface modes. The simulation spectrum is obtained on the optical axis after propagation by $38 \mathrm{~mm}$. The position of the dip and shape of the hump near $1.5 \mathrm{THz}$ are very sensitive to the value of $h$ which varies by $\sim 10 \%$ across the sample because of nonuniformity in the resist coating process. 

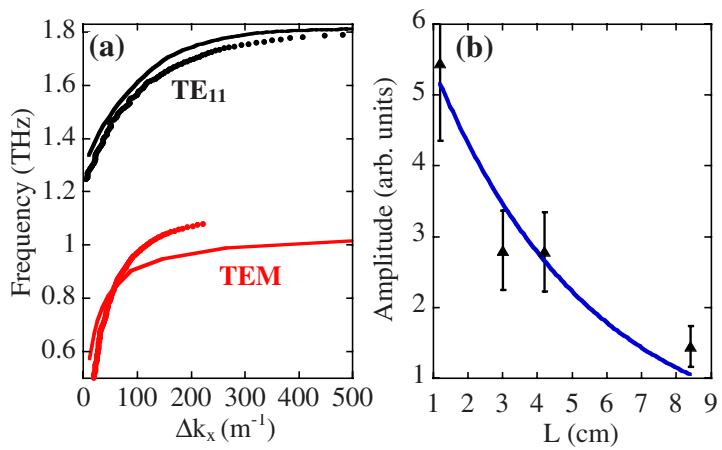

FIG. 4. (Color online) (a) Deviation of SSPP dispersion curves from the light line. Points are from experiment, continuous curves are calculated using the CMM. (b) Measured square root of $\mathrm{THz}$ pulse energy vs propagation length. The solid curve is an exponential fit to describe the attenuation.

Measurement of the phase spectrum $\phi(\omega)$ corresponding to the amplitude spectrum in Fig. 3(a) allows the dispersion relation to be obtained using the equation $k_{x}(\omega)=k_{o}$ $+\Delta \phi(\omega) / L$. Here $k_{o}=\omega / c$ is the free space wavevector, $\Delta \phi$ is the difference between the phase spectra with and without the sample in place, and $L$ is the sample length. Figure 4(a) shows the measured difference between $k_{x}$ and $k_{o}, \Delta k_{x}$, together with that calculated using the CMM. The agreement between calculation and experiment for the $\mathrm{TE}_{11}$ mode is excellent. The noticeably worse agreement for the TEM mode is probably due to the variation in $h$ across the sample. The experimental dispersion results shown in Fig. 4(a) allow us to estimate the out of plane extent of the guided waves. For the TEM mode at $0.90 \mathrm{THz}$, where the signal is a maximum, we estimate the exponential field decay length, $L_{z}=1 / \sqrt{ }\left(k_{x}^{2}-k_{o}^{2}\right)$, to be $620 \pm 40 \mu \mathrm{m}(\sim 2 \lambda)$. For the $\mathrm{TE}_{11}$ mode at $1.50 \mathrm{THz}$ we obtain $L_{z}$ $=500 \pm 50 \mu \mathrm{m}(\sim 2.5 \lambda)$. These values are a hundred times smaller than expected for a fully evolved SPP on a flat copper surface, known as a Zenneck wave. ${ }^{20}$ The narrow frequency window where the group velocity of the TEM mode is very small and over which $L_{z}$ theoretically approaches $\lambda / 8$ at the band edge, is not accessible in our measurements. This is because of poor field overlap with the $\mathrm{THz}$ beam, higher attenuation, and the broadband nature of time domain spectroscopy.

Figure 4(b) shows the square root of the detected pulse energy, measured as a function of sample length. The pulse energy is estimated by squaring and integrating the time domain traces. The longest sample consists of two $42 \mathrm{~mm}$ long pieces accurately butted together. The error bars reflect the reproducibility in positioning the sample and cylindrical lenses. An exponential fit to the data yields a frequency averaged amplitude attenuation coefficient of $\alpha$ $=0.22 \pm 0.06 \mathrm{~cm}^{-1}$. For comparison, we modeled the pulse propagation using the finite integration technique in a $30 \mathrm{~mm}$ long simulation volume extending $5 \mathrm{~mm}$ above the metamaterial surface with perfectly absorbing boundary conditions at the upper surface and periodic boundary conditions transverse to the propagation direction. The finite metal conductivity was incorporated using surface impedance boundary conditions, which provide accurate results for metals with large permittivities. ${ }^{21}$ In a similar way to the experiment, we monitored the decay of the electric field as a function of time at different points along a propagation axis $100 \mu \mathrm{m}$ above the surface. By comparing the results for a copper conductivity equal to the bulk value of $5.8 \times 10^{7} \Omega^{-1} \mathrm{~m}^{-1}$ with the perfect electrical conductor case, we were able to infer an Ohmic contribution to $\alpha$ of $0.05 \pm 0.01 \mathrm{~cm}^{-1}$. The higher value of the experimental attenuation coefficient can probably be attributed to a combination of lower than assumed metal conductivity and scattering by the sample boundaries and imperfections. While the loss is not negligible, there is an acceptable tradeoff with confinement for the fewcentimeter-scale devices that might be envisaged in practical applications such as sensing.

In summary, we have presented experimental results and supporting numerical simulations showing propagation of tightly bound and relatively low loss SSPP-THz waves on a metamaterial surface containing annular cavities. Guiding in two independently variable and relatively low loss frequency bands can be supported. Together with efficient end-fire coupling, this could be useful in waveguide-based chemical or biochemical sensing where the ability to ratio responses in at least two distinct frequency bands with high dynamic range is desirable for quantitative analysis.

We acknowledge the financial support of the Air Force Office of Scientific Research and the Spanish Ministry of Science under Projects Nos. MAT2008-06609-C02 and CSD2007-046-Nanolight.es. We thank Byron Alderman and David Wilby for the copper sputtering and wafer dicing.

${ }^{1}$ M. Tonouchi, Nat. Photonics 1, 97 (2007).

${ }^{2}$ D. Grischkowsky, S. Keiding, M. van Exter, and Ch. Fattinger, J. Opt. Soc. Am. B 7, 2006 (1990).

${ }^{3}$ R. M. Woodward, B. E. Cole, V. P. Wallace, R. J. Pye, D. D. Arnone, E. H. Linfield, and M. Pepper, Phys. Med. Biol. 47, 3853 (2002).

${ }^{4}$ C. J. Strachan, P. F. Taday, D. A. Newnham, K. C. Gordon, J. A. Zeitler, M. Pepper, and T. Rades, J. Pharm. Sci. 94, 837 (2005).

${ }^{5}$ R. Piesiewicz, T. Kleine-Ostmann, N. Krumbholz, D. Mittleman, M. Koch, J. Schoebel, and T. Kurner, IEEE Antennas Propag. Mag. 49, 24 (2007).

${ }^{6}$ K. Yamamoto, M. Yamaguchi, F. Miyamaru, M. Tani, M. Hangyo, T. Ikeda, A. Matsushita, K. Koide, M. Tatsuno, and Y. Minami, Jpn. J. Appl. Phys., Part 2 43, L414 (2004).

${ }^{7}$ M. Nagel, P. Haring Bolivar, M. Brucherseifer, and H. Kurz, Appl. Phys. Lett. 80, 154 (2002).

${ }^{8}$ M. Nagel, F. Richter, P. Haring Bolivar, and H. Kurz, Phys. Med. Biol. 48, 3625 (2003).

${ }^{9}$ J. Zhang and D. R. Grischkowsky, Opt. Lett. 29, 1617 (2004).

${ }^{10}$ H. Hirori, K. Yamashita, M. Nagai, and K. Tanaka, Jpn. J. Appl. Phys., Part 2 43, L1287 (2004).

${ }^{11}$ C. R. Williams, S. R. Andrews, S. A. Maier, A. I. Fernandez-Dominguez, L. Martin-Moreno, and F. J. Garcia-Vidal, Nat. Photonics 2, 175 (2008).

${ }^{12}$ J. B. Pendry, L. Martin-Moreno, and F. J. Garcia-Vidal, Science 305, 847 (2004).

${ }^{13}$ F. I. Baida and D. Van Labeke, Phys. Rev. B 67, 155314 (2003).

${ }^{14}$ W. Fan, S. Zhang, B. Minhas, K. J. Malloy, and S. R. J. Brueck, Phys. Rev. Lett. 94, 033902 (2005)

${ }^{15}$ SU-8 photoresist, MichroChem Corp., Newton, MA, USA. www.michrochem.com.

${ }^{16}$ N. Marcuvitz, Waveguide Handbook (McGraw-Hill, New York, 1951).

${ }^{17}$ J. Bravo-Abad, F. J. Garcia, and L. Martin-Moreno, Phys. Rev. Lett. 93 227401 (2004).

${ }^{18}$ J. Saxler, J. Gomez-Rivas, C. Janke, H. P. Pellemans, P. Haring-Bolivar, and H. Kurz, Phys. Rev. B 69, 155427 (2004).

${ }^{19}$ CST Microwave Studio, Computer Simulation Technology, Wellesley Hills, MA, USA. www.cst.com.

${ }^{20}$ T.-I. Jeon and D. R. Grischkowsky, Appl. Phys. Lett. 88, 061113 (2006).

${ }^{21}$ M. A. Leontovich, Investigation of Propagation of Radio Waves, Part II (USSR Acad. Sci, Moscow, 1948). 